\title{
Dangereuse Aspirine
}

La nocivité de Гaspirine pour les ulcéreux n'est plus discutée aujourd'hui, ni son role hémorragipare sur les muqueuses digestives (voir Editorial: «Aspirine et hémorragie digestive»; dans ce journal vol. 91, p. 274, 1959). Mais les avis restent partagés quant au méca-nisme de cette action. Schématiquement, on a invoqué les pathogénies suivantes:

$1^{\circ}$ Effet local, irritant et corrosif; théorie appuyée par les pro-priétés épithéliolytiques des salicylés; par les brûlures gastriques ac-compagnant les manifestations d'intolérance; par les constatations gastroscopiques répétées depuis les premieres observations de Douth-waíthe et Líntott en 1938.

$2^{\circ}$ Reaction de type allergíque, suggérée par Гindividualité des accidents cliniques qui ne correspondent pas à la dose reçue ou à la durée du traitement. Combinée à Гélément toxique local, elle consti-tue faction toxo-allergíque de Levrat.

$3^{\circ}$ Action «steroíd-like», c'est-à-dire activité pharmacologique évo-quant celle des hormones de l'écorce surrénalienne, par Гintermédiaire du relai corticotrope (ACTH) de Thypophyse, Tinfluence de l'aspirine sur le diencéphale n'étant pas mise en doute. D'autre part, les salicylés possèdent un pouvoir anti-inflammatoire analogue à celui de la cortisone ou de la phénylbutazone (cette dernière cependant lèse la mu-queuse gastrique par voie humorale, en produisant une necrose directe des cellules nobles sans phénomènes inflammatoires, au lieu de la reaction d'hyperémie déclenchée par Гaspirine).

$4^{\circ}$ Facteur humoral, un taux élevé de salicylémie prédisposant à Гérosion gastrique. Cependant ces faits ne peuvent pas être interprétés comme la consequence d'une excretion, par Гestomac, du produit injecté dans les veines. Ici les conditions expérimentales s'éloignent de Tobservation clinique, d'abord par Гusage exceptionnel des salicylés par voie parentérale, d'autre part à cause d'un dosage très supérieur chez Гanimal (dans les recherches qui vont suivre, 0,47 à 1,9 gramme pour un chat de 2 à $4 \mathrm{~kg}$ représenteraient entre 10 et 50 grammes pour un adulte de $60 \mathrm{~kg}$ !) $5^{\circ}$ Uinfluence de Taspírine sur la secretion gastrique est le point le plus controversé. Les premiers travaux allemands, il y a plus de 30 ans, avaient fait admettre comme un fait evident la stimulation

370

Editorial

marquee de la production d'acide dans Гestomac; plus tard, le groupe anglais qui décrivit Faction hémorragipare de $\Gamma$ aspirine, nia son influence hypersecrétoire; enfin, plus récemment, Cravati et Cosgrove (1946), Winkelman et Summerskill (1961) ont même soutenu le role inhî́biteur de Vaspirine sur la secretion gastrique.

A ce sujet, un important travail experimental provenant de la Clinique chirurgicale de Sydney, vient d'etre publié (Gut, vol. 5, pp. 230-236, juin 1964), qui ouvre des aperçus nouveaux sur ces pro-blèmes; Annette Lynch, Helen Shaw et G. W. Milton ont examine dans diverses conditions la secretion de la poche gastrique isolée et énervée de 33 chats recevant de l'aspirine, avant stimulation histaminique endoveineuse (2,2 $\mathrm{mg}$ par $\mathrm{kg}$ et par minute pendant 6 heures), et de 19 témoins. 


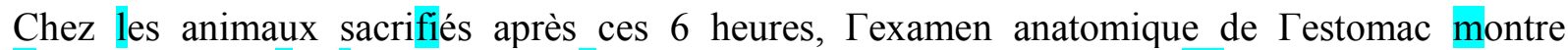
Iabsence oVulcération dans les series I et III qui ne reçurent pas a"aspirine (même après histamine à dose supra-maximale; série III). En revanche, chez tous les chats recevant de Taspirine, les lesions allaient de quelques hémorragies sous-muqueuses à des erosions innombrables; elles étaient réparties sur tout le corps de Гestomac, sauf sur $\Gamma$ antre prépylorique (pylore toujours ligature). Histolo-giquement, on observait un aplatissement des cellules cuboïdes, mais pas de desintegration des cellules principales ou pariétales, comme si Vepithelium avait été abrasê par place, le reste de la muqueuse étant intact.

$\mathrm{Du}$ point de vue biologique, chez les animaux non-stímulês antê-rieurement par Thistamine (series I et II), Гaspirine insoluble (1,9 g; série II) réduit à presque rien le volume sécrété dès la $2^{\circ}$ heure, alors que les témoins (série I) ne varient guère; de même la concentration acide devient nulle dès $3 \%$ heures dans la série II («aspirinée») alors qu'elle baisse à peine dans la série I (témoin).

Aux doses indiquées, Thistamine (série III) excite une secretion de 91,8 $\pm 21,9 \mathrm{ml}$ en 6 heures, avec un debit acide de 13,0 $\pm 3,7 \mathrm{mEq}$. Avec Гaspirine, soluble ou insoluble, (series IV à VIII), le volume varie entre $16,1 \pm 7,8$ et $47,4 \pm 19,3 \mathrm{ml} / 6 \mathrm{~h}$., et le debit entre $0,7 \pm 0,3$ et $69 \pm 2,7$ mEq.

En conclusion, Vaspírine dêprime - de façon statistiquement significative - la secretion gastrique $d u$ chat, ceci proportionnellement à la quantité d'aspirine introduite préalablement $(0,47,0,95$ ou $1,9 \mathrm{~g})$; d'autre part, la forme soluble du medicament inhibe moins la secretion gastrique que la forme insoluble.

Editorial

371

Dans une série IX, la drogue fut introduite dans le duodenum (le pylore était ligature dans les 9 series). Les lesions anatomiques de $\Gamma$ estomac se superposaient parfaitement aux autres, alors que Tintestin grêle était peu altéré; quant à $\Gamma$ effet freinateur sur la secretion, il équivalait celui de Taspirine introduite directement dans Testomac.

Ces observations expérimentales permettent aux auteurs de prendre position vis-à-vis des theories pathogéniques rappelées au debut de cet editorial. Elles excluent les mécanismes vasculaire, allergique et sur-tout stéroïde: la cortisone en effet excite la secretion gastrique au lieu de la déprimer, et le relai hypothalamico-surrénalien ne joue pas chez un animal profondément anesthésié. Et elles retiennent, d'une part Гỉnfluence nêfaste du contact de Гaspirine sur la muqueuse gastrique, spécifiquement sensible à cette drogue, puisqu'on trouve

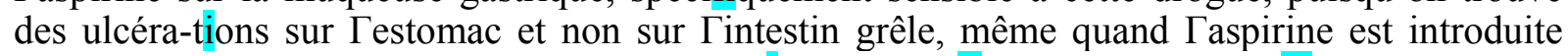
dans ce dernier; d'autre part, le taux de salícylês círcu-lant possède également un pouvoir gastrotrope nocif. A. Lynch et $H$. Shaw se font done les partisans résolues de Faction toxíque dîrecte sur la lumière gastrique de Vaspirine ou de ses derives.

Toutes reserves faites sur la question des doses utilisées (v. plus haut), ces recherches entraînent deux conclusions pratiques:

$1^{\circ}$ L'aspirine ne devrait jamais être administrée à dose élevée sans être associée - ou mieux: précédée - par un pansement gastrique (mais non par un antacíde neutralisant chimiquement ГHC1!).

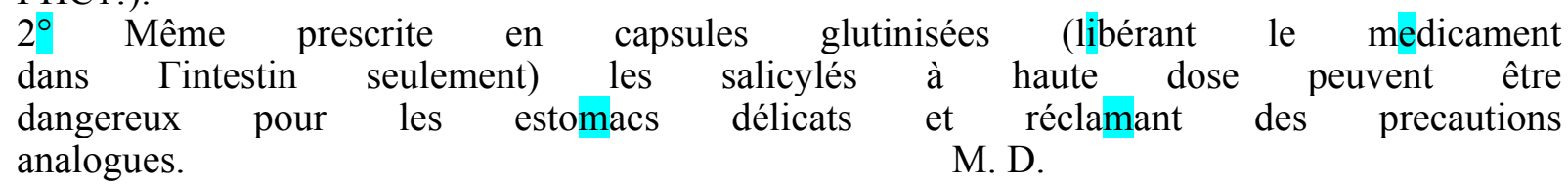


26 Gastroenterologia, Voḷ. 101, No. 6 (1964) 